\title{
Wer ernährt die Familie? Erwerbs- und Einkommenskonstellationen in Ostdeutschland
}

\author{
Christina Klenner
}

Zwanzig Jahre sind vergangen seit dem historischen Wendepunkt 1989, der nicht nur die politische Landkarte grundlegend veränderte, sondern auch das Leben ostdeutscher Frauen und Männer umkrempelte. Das ist Anlass, mit Blick auf die Geschlechterverhältnisse eine Bilanz des Transformationsprozesses zu ziehen. Anhand neuer Daten wird gezeigt, dass es zu der befürchteten Re-Traditionalisierung in Ostdeutschland bisher nicht gekommen ist. Ostdeutschland hat das männliche Familienernährermodell nicht übernommen; es ist insgesamt weiter auf dem Rückzug. Im Gegenzug nimmt die Bedeutung des Erwerbseinkommens von Frauen für die Existenzsicherung zu: Zweiverdienerpaare dominieren und der Anteil an Familienernährerinnen wächst.

\section{Fragestellung}

Wenn es bei der deutschen Vereinigung 1990 hieß „Ein Staat - zwei Gesellschaften“, so galt das ganz besonders für die Geschlechterverhältnisse. Den Frauen aus Ostdeutschland wurde Anfang der 1990er Jahre ein „Gleichstellungsvorsprung“ gegenüber westdeutschen Frauen attestiert (Geißler 1993). Zum einen, weil im Osten mehrheitlich andere Geschlechterarrangements gelebt wurden als im Westen. Der Übergang hin zu neuen Leitbildern und Geschlechterrollen jenseits des männlichen Ernährermodells (Leitner et al. 2004) hat im Osten Deutschlands bereits mehr als eine Generation früher begonnen. Deswegen verstanden sich Frauen beider Teile Deutschlands bezüglich ihrer Lebensentwürfe und Auffassungen zu Arbeit und Familie oftmals kaum, die Rede war von „Stiefschwestern" (Rohnstock 1994).

Zum anderen hatte sich im Osten Deutschlands auch die Geschlechterhierarchie etwas abgeflacht (Dölling 2005). ${ }^{1}$ Das zeigen Kenngrößen wie Erwerbsquoten, Arbeitszeitdifferenzen und Gender Pay Gap (geschlechtsspezifische Lohnungleichheit), die zur Messung der Gleichstellung herangezogen werden (EC 2009; Bothfeld et al. 2005). Damit wurde auch die soziale Ungleichheit zwischen den Geschlechtern etwas abgebaut.

Jahrelang sind immer wieder Befürchtungen geäußert worden, dass es infolge des Transformationsprozesses, dessen soziale Kosten auch viele Frauen zu tragen hatten und haben, sowie durch den Institutionentransfer bei der deutschen Vereinigung $\mathrm{zu}$ einer Re-Traditionalisierung der Ge- schlechterverhältnisse kommen würde. Dass sich nach einer kurzen Übergangsperiode das typische westdeutsche Geschlechtermodell infolge der Übertragung der politischen Institutionen auf den Osten auch dort durchsetzen würde, war ein denkbares Szenario. Zumindest galt es als wahrscheinlich, dass der ostdeutsche Geschlechtervertrag erheblichen Spannungen ausgesetzt sein würde, weil die Vorstellungen über Geschlechterrollen bei Frauen und Männern fortan stärker auseinanderdriften könnten. Als möglich erschien, dass Männer es infolge ihrer eigenen sozialen Verunsicherung vielleicht begrüßen könnten, wenn Frauen vom Arbeitsmarkt verdrängt werden, während die Frauen dagegen auf ihrer Erwerbsteilhabe beharren würden (Schenk 2003, S. 63). Als eher unwahrscheinlich galt das Szenario, dass das ostdeutsche Modell seine Bedeutung behält und zwischen Frauen und Männern weiterhin ein Konsens über die Arbeitsmarktteilhabe von Frauen besteht. Der Rückblick zeigt jedoch: Die ostdeutschen Frauen haben sich mit ihrem „Eigensinn" dem neuen Institutionensystem nicht angepasst (Dölling 2005). Damit hat sich die Re-Traditionalisierungsthese in ihrer Undifferenziertheit nicht bewahrheitet.

Im Mittelpunkt des Beitrages steht die Frage, wie weit die Abkehr vom Modell des männlichen Familienernährers im Osten Deutschlands vorangeschritten ist, welche Erwerbskonstellationen und Einkommensrelationen im Paarzusammenhang sich hier entwickelt haben und ob sich in Teilsegmenten der Gesellschaft doch traditionelle Geschlechterarrangements reproduziert haben. Zunächst wird das für den Osten Deutschlands typische Geschlechtermodell $^{2}$ charakterisiert und untersucht, inwieweit dieses vorherrschende Modell im Transformationsprozess erhalten geblieben ist. Anhand der wichtigsten Kennzeichen des Modells werden Persistenz und Wandel dargestellt (Abschnitt 2). Die Argumentation wird durch eine Datenanalyse auf Basis des Sozio-oekonomischen Panels (SOEP) gestützt (Abschnittte 3 bis 5). Insoweit werden Zwischenergebnisse eines noch laufenden Forschungsprojektes „Flexible Familienernährerinnen " vorgestellt, in dessen Rahmen neue Geschlechterarrangements mit quantitativen und qualitativen Methoden erforscht werden. ${ }^{3}$ Abschnitt 6 zieht ein Fazit und verweist auf offene Fragen.

1 Zur Analyse der ostdeutschen Geschlechterverhältnisse vgl. auch u. a. Nickel 1993; Trappe 1995; Gensior 1995; Budde 1997; Kolinsky/Nickel 2003; Völker 2004; Schäfer et al. 2005.

2 Der verwendete Begriff "Geschlechtermodell “ umfasst nicht nur das "Geschlechterregime“ , das sich auf ein Set an Nomen, Werten, Politiken, Prinzipien und Gesetzen bezieht, das die Beziehungen der Geschlechter beeinflusst (vgl. Mac Rae 2006, S. 224f., zit. bei Betzelt 2007, S. 11f., Übersetzung C. K.), sondern der Begriff "Geschlechtermodell" erfasst darüber hinaus die am meisten verbreiteten typischen Praktiken der Arbeitsteilung und Identitätsbildung von Männern und Frauen, für die auch der Begriff "Geschlechterarrangement" (Dölling 2005) verwendet wird.

3 Unter Leitung der Verfasserin wird derzeit das WSI-Projekt „Flexible Familienernährerinnen. Prekarität im Lebenszusammenhang von ostdeutschen Frauen?" unter Mitarbeit von Petra Drauschke (bis 12/08) und Svenja Pfahl (SoWiTra Berlin, ab 4/09) durchgeführt. Ein parallel angelegtes, von der Hans-Böckler-Stiftung finanziertes Projekt läuft unter Leitung von Ute Klammer an der Universität Duisburg-Essen unter Mitarbeit von Sabine Neukirch und Dagmar Weßler-Poßberg und bezieht sich auf das empirische Feld Westdeutschland.

Christina Klenner, Dr., Wissenschaftlerin im WSI in der Hans-Böckler-Stiftung. Arbeitsschwerpunkte: Frauenerwerbstätigkeit, Geschlechterverhältnisse, work-life Balance. e-mail: christina-klenner@boeckler.de 


\section{Das extensive Zweiverdienermodell in Ostdeutschland}

\subsection{CHARAKTERISTIKA DES MODELLS}

Seit Ende der 1960er Jahre setzte sich in der DDR allmählich immer stärker ein $\mathrm{Ge}$ schlechtermodell durch, das sich als „Doppel-Versorger-Modell“ (Dölling 2003) oder spezieller als vergeschlechtlichtes extensives Zweiverdienermodell (Klenner/Hašková 2009) bezeichnen lässt. Beide Partner sind dabei weitgehend kontinuierlich erwerbstätig. Unterbrechungen für Familienphasen wurden im Lauf der Zeit immer kürzer (Trappe 1995). Extensiv ist das Modell wegen der hohen Bedeutung der Erwerbsarbeit auch für Frauen, die häufig in Vollzeit und mit langen Arbeitszeiten außer Haus arbeiten. Als vergeschlechtlicht ist es zu kennzeichnen, weil die Teilhabe von Frauen am Arbeitsmarkt nicht zur beruflichen Gleichstellung führt und auch die Aufteilung der unbezahlten Arbeit traditionellen geschlechtsspezifischen Mustern weiter folgt (Nickel 1993; Kolinsky 2003).

Diese wesentlichen Kennzeichen des Modells werden im Folgenden kurz erläutert und es wird gezeigt, inwieweit sie im Laufe der letzten 20 Jahre erhalten geblieben sind.

(1) Das ostdeutsche Geschlechtermodell war und ist in hohem Maße erwerbszentriert und auf eigenständige Existenzsicherung - auch von Frauen - durch Erwerbsarbeit ausgerichtet. Erwerbstätigkeit - vor 1989 Berufstätigkeit genannt - hatte für Frauen ebenso wie für Männer doppelte Bedeutung: als sinnerfüllte Tätigkeit und zentraler gesellschaftlicher Integrationsmodus sowie als Basis des Einkommenserwerbs. Wenn auch nicht alle Frauen aufgrund ihrer Einkommenshöhe vollständig unabhängig vom Einkommen des männlichen Partners waren (Trappe/Sørensen 2005), so hatten sie doch eine relativ ausgeprägte Autonomie, weil die meisten Einkommen Existenz sichernd waren und die berufliche Einbindung gewährleistet war. Männer akzeptierten spätestens ab den 1970er Jahren die berufliche Tätigkeit der Frauen als Selbstverständlichkeit (Scholz 2005, S. 142). Der Kern des Modells, dass grundsätzlich alle erwachsenen Frauen und
Männer ihre Existenz durch eigene Erwerbsarbeit sichern (sollen), wird heute als Anspruch immer noch von den meisten geteilt (Schmidtke 2004, S. 298f.). Damit entspricht es weitgehend dem Leitbild des „adult worker model“ („Zwei-Erwerbstätigen"-Familie, vgl. Lewis 2004), das die europäische Politik zunehmend prägt. Auch in der Realität ist die Erwerbsquote ostdeutscher Frauen - obwohl sie im Transformationsprozess zurückgegangen ist mit 74,7 \% (2008) nach wie vor hoch. Sie liegt nur wenig, um rund sieben Prozentpunkte, unter der von ostdeutschen Männern (81,6 \%). Aufgrund der Arbeitslosigkeit ist allerdings die tatsächliche Beschäftigungsquote (Erwerbstätigenquote) von Frauen deutlich niedriger $(64,9 \%)$ (StBA 2009b). 4

(2) In Ostdeutschland war und ist das Leitbild der berufstätigen Mutter vorherrschend (Kolinsky/Nickel 2003; Wagner et al. 2006). Wie Kindheit konstruiert ist - und damit verbunden, welche Form der Kinderbetreuung als richtig angesehen wird -, ist ein wesentliches Element der jeweiligen Geschlechterarrangements (Pfau-Effinger 1998). Beim traditionellen Ernährermodell gilt Kindheit als privat, eine Ansicht, die im Westen nach wie vor Prägekraft hat. Im Unterschied dazu ist in Ostdeutschland Kindheit vorwiegend öffentlich konstruiert, wie es dem Doppel-Versorger-Modell mit staatlicher Kinderbetreuung (Pfau-Effinger 2000, S. 88) entspricht. Daher ist es kulturell akzeptiert, dass beide Eltern arbeitsmarktaktiv sind und Kinder in öffentlichen Einrichtungen betreut werden. Überwiegend ist dies heute noch der Fall, da das Netz von Kindertageseinrichtungen in Ostdeutschland in den Grundzügen erhalten geblieben ist.

(3) Das ostdeutsche Geschlechtermodell ist durch eine hohe Vollzeitorientierung von Frauen gekennzeichnet. Teilzeitarbeit spielt als spezifisch weibliche Form von Erwerbstätigkeit eine geringere Rolle als in Westdeutschland. 1989 betrug die Teilzeitquote in der DDR $27 \%$ (Klammer et al. 2000, S. 168), und sie stieg - nach einem transformationsbedingten Rückgang - bis 2007 auf $35 \%$ (StBA 2009b). Insbesondere Minijobs sind im Osten weit weniger verbreitet als im Westem (StBA 2009c). Mehr als die Hälfte der Teilzeitarbeitsverhältnisse sind in Ostdeutschland arbeitsmarktbedingt und unfreiwillig (StBA 2009a). Auch hierin schlägt sich nieder, dass Ostfrauen eine eigenständige Existenzsicherung anstreben und die Absicherung über die Ehe, wie sie für einen beträchtlichen Teil der westdeutschen Frauen besteht, im Osten kaum akzeptiert wird.

(4) Als weiteres Charakteristikum des ostdeutschen Geschlechtermodells ist anzusehen, dass es einerseits die berufliche und soziale Ungleichheit der Geschlechter nicht aufzuheben vermochte, was sich unter anderem in der geringen Repräsentanz von Frauen in Führungspositionen, im Gender Pay Gap oder dem Mangel an Freizeit von Frauen niederschlug (Nickel 1993), andererseits ostdeutsche Frauen aber ein Mehr an Gleichstellung erreicht haben. Am Ende der DDR wiesen die Kenngrößen eine stärker angeglichene Situation aus als in Westdeutschland. Auch dieser Aspekt - die vorhandene, aber abgeflachte Hierarchie zwischen Männern und Frauen - ist bis heute erhalten geblieben. So ist die geschlechtsspezifische Einkommenslücke in Ostdeutschland kleiner als in Westdeutschland (Bothfeld et al. 2005, S. 262f.; Bispinck et al. 2009). 5 Bezüglich der Teilung der unbezahlten Familienarbeit zwischen Männern und Frauen hat sich im Osten Deutschlands nicht viel verändert, aber auch hier sind ostdeutsche Männer etwas stärker an der unbezahlten Arbeit beteiligt als ihre Geschlechtsgenossen in Westdeutschland (Gille/Marbach 2004, S. 96; Scholz 2005).

\section{2 ÜBERLEBENSKRAFT DES MODELLS}

In seinen Kernpunkten besteht das vorherrschende ostdeutsche Geschlechtermodell also nach wie vor. Offen ist, ob das Modell den Generationenwechsel überleben wird, da allmählich die Frauen und Männer, die es verinnerlicht hatten, aus dem Erwerbsalter herauswachsen. Man könnte annehmen, dass Individualisierung und Pluralisierung zu einem stärker ausdifferenzierten Verhalten unter jüngeren Frauen und Männern führen. Es wäre nicht verwunderlich, wenn die Jüngeren teilweise traditionellere Vorstellungen entwickeln,

\footnotetext{
4 Ich danke Katrin Menke für ihre Mitarbeit bei den Datenrecherchen.

5 Die Werte schwanken je nach Datenquelle und erfasstem Personenkreis erheblich, der Ost-WestUnterschied schlägt sich aber in allen Statistiken nieder (Bothfeld et al. 2005, S. 262f.).
} 
da ihre Orientierungen in einem anderen gesellschaftlichen Umfeld geprägt werden und sie von den Anreizwirkungen des deutschen Steuer- und Sozialsystems beeinflusst werden. Diese Systeme privilegieren die Ehe und die daraus abgeleitete Existenzsicherung von verheirateten Frauen. Eine Sogwirkung wäre plausibel ist aber kaum ersichtlich. Auf der Ebene der Einstellungen lässt sich ein solcher Wandel nicht nachweisen. So hat nach der ALLBUS-Umfrage der „Anteil moderner Äußerungen "6 in Ostdeutschland zwischen 1991 und 2004 um 19 Prozentpunkte zugelegt (auf $86 \%$ ) und liegt in der Altersgruppe der 18- bis 30-Jährigen mit $92 \%$ extrem hoch und über den Werten aller anderen Altersgruppen $^{7}$ (StBA 2006, S. 518). Ein anderes Kennzeichen des Beharrens auf dem bisherigen Anspruch ist die Migrationsbereitschaft von ostdeutschen Frauen. Um einen Ausbildungs- oder Arbeitsplatz zu erhalten, gehen zahlreiche ostdeutsche Frauen in andere Landesteile (Bothfeld et al. 2005, S. 38f.; Kröhnert/Klingholz 2007). Dass das Verständnis, für sich selber zu sorgen, bei den ostdeutschen Frauen tief verankert ist, zeigt sich auch an der Einstellung zur Ehe. Sie gilt im Osten kaum als die typische Form der Absicherung von Mutter und Kind(ern). Mit 60,7 \% wurden 2008 in Ostdeutschland weit mehr als die Hälfte aller Kinder von ledigen Müttern geboren (im Westen sind es 25,8 \%, StBA 2009d). Das könnte als der Wille ostdeutscher Frauen zu interpretieren sein, vom Partner in ökonomischer wie rechtlicher Hinsicht unabhängig zu bleiben.

„Kontinuität trotz Transformation“ zu diesem Ergebnis kam auch eine qualitative Studie, die zeigt, dass die Ernährerrolle des Mannes und die Hausfrauenrolle der Frau von den Befragten klar abgelehnt wurde (Wagner et al. 2006). Der ganzheitliche Lebensentwurf, der „Doppelanspruch“ von Frauen, wonach Frauen weder nur Hausfrau sein noch zugunsten der Erwerbsarbeit auf Kinder verzichten wollen, wird nach wie vor von Ostdeutschen mehrheitlich geteilt.

Neben Beharrung in wesentlichen Aspekten zeigen sich aber auch Veränderungen, deren künftige Wirkungen auf die Geschlechterverhältnisse noch nicht absehbar sind. So steigen nicht nur die Teilzeitquoten ostdeutscher Frauen kontinuierlich an, sondern auch die Orientierung auf Teilzeitarbeit bei Müttern hat zugenommen (Thelen/Baerwolf 2007; Beckmann
2002). Auch sind junge ostdeutsche Frauen stärker von geschlechtsspezifischen Einkommensdifferenzen betroffen als ältere, was auf sich wieder verstärkende berufliche Ungleichheiten hindeutet. Der Gender Pay Gap bei Berufseinsteigerinnen ist im Osten zwar etwas geringer als im Westen, mit $17,8 \%$ in den ersten drei Jahren nach Berufseinstieg aber gleichwohl hoch. Den geringsten Einkommensabstand haben ältere Frauen mit mehr als zehn Jahren Beschäftigung (14,7 \%) (Bispinck et al. 2009).

Insgesamt zeigt sich, dass das ostdeutsche Geschlechtermodell bisher sowohl auf der faktischen Ebene als auch auf der Ebene der Einstellungen in seinen Grundzügen erhalten geblieben ist. Veränderungen haben sich bislang ohne starke statistische Ausschläge - quasi „unter der Oberfläche“ - vollzogen, sodass sich noch nicht sagen lässt, ob sie möglicherweise doch als Vorboten einer generelleren Veränderung gedeutet werden müssen.

\section{Erwerbskonstellationen
und Einkommensrelationen in Paarhaushalten}

Im Folgenden wird untersucht, ob auf der Ebene der Erwerbskonstellationen von Paaren und der Einkommensrelationen im Haushalt Verschiebungen im Laufe des Transformationsprozesses stattgefunden haben, und wenn ja, in welche Richtung. Auch hierin könnte sich eine Veränderung oder Ausdifferenzierung von Geschlechterarrangements zeigen. Unklar ist, welche Bedeutung vor dem Hintergrund tief greifender Wandlungsprozesse in der Sozialstruktur die eigenständige Existenzsicherung für Frauen und Männer in Ostdeutschland hat. Wo gibt es weiterhin oder erneut eine ökonomische Abhängigkeit der Frauen von ihrem Partner? Wir haben da$\mathrm{zu}$ auf der Basis des Sozio-oekonomischen Panels (SOEP) Familien- und Paarhaushalte analysiert. ${ }^{8}$ Überprüft werden sollte, inwieweit sich Re-Traditionalisierungsprozesse auf der Paarebene zeigen oder nicht zeigen. Einerseits könnte - so die Ausgangsüberlegung - Arbeitslosigkeit und prekäre Beschäftigung von Frauen deren Einkommensposition schwächen und Männer wieder verstärkt in die Ernährerrolle drängen. Andererseits sind aber auch Männer von den Problemen am Arbeitsmarkt betroffen und zudem könnten Frauen durch den Wechsel der Geschlechtertypik von Berufen sowie veränderte Arbeitsbewertungen (z. B. bei Finanzdienstleistungen) eine Aufwertung ihrer Erwerbsposition im Paarzusammenhang erfahren. So haben z.B. bereits Nickel (2003) sowie Völker (2004) darauf hingewiesen, dass ostdeutsche Frauen aufgrund der Umbrüche am Arbeitsmarkt teilweise die Rolle als „Ernährerinnen ihrer Familie“ übernommen haben.

\subsection{ERWERBSKONSTELLATIONEN}

Die Erwerbskonstellationen im Paarzusammenhang zeigt Tabelle $1^{9}$ : Dass beide Partner erwerbstätig sind, ist in Ostdeutschland nach wie vor die Regel. Die große Mehrheit der Paare sind Zweiverdienerpaare (72\%, 2007). Dies kommt heute zwar um elf Prozentpunkte seltener vor als 1990, aber rechnet man die Haushalte mit einem arbeitslosen Partner (oder einer Partnerin) hinzu, dann ist die Konstellation, dass beide auf den Arbeitsmarkt hin orientiert sind, heute genauso häufig wie 1990 (83\%). Zweiverdienerpaare sind in Ostdeutschland trotz der Zunahme von unfreiwilligen Einverdienerhaushalten, in denen ein Partner arbeitslos ist, stärker verbreitet als in Westdeutschland. ${ }^{10}$

6 Die Zuordnung einzelner Frageformulierungen zu Einstellungen zur Rolle der Frau wurde von den Autorinnen und Autoren des "Datenreport 2006" (StBA 2006, S. 517) vorgenommen. Modern ist beispielsweise die Zustimmung zur Formulierung: „Eine berufstätige Mutter kann ein genauso herzliches und vertrauensvolles Verhältnis zu ihren Kindern finden wie eine Mutter, die nicht berufstätig ist ${ }^{\prime}$; oder die Ablehnung des Statements: „Eine verheiratete Frau sollte auf eine Berufstätigkeit verzichten, wenn es nur eine begrenzte Anzahl von Arbeitsplätzen gibt, und wenn ihr Mann in der Lage ist, für den Unterhalt der Familie zu sorgen“.

7 Die Vergleichswerte für Westdeutschland liegen im Durchschnitt bei $68 \%$ und bei $86 \%$ bei den 18 bis 30-Jährigen.

8 Es handelt sich beim gegenwärtigen Stand der Projektbearbeitung um zwei getrennte Auswertungen des SOEP, sodass die Analysejahre differieren. Ein WSI-Diskussionspapier von Brehmer/Klammer/ Klenner, das die Analysen fortführt, ist in Vorbereitung.

9 Die Werte bis 2003 finden sich auf der CD-Rom des WSI-FrauenDatenReports 2005 (Bothfeld et al. 2005). Für 2007 hat mein Kollege Wolfram Brehmer die Berechnungen durchgeführt, wofür ich ihm herzlich danke.

10 In Westdeutschland sind heute bei $66 \%$ der Paare beide Partner erwerbstätig, wobei der Zuwachs vor allem in den letzten Jahren erfolgte (Vergleichszahl für 1990: $49 \%$, vgl. Tabelle 1). 
Die Erwerbskonstellation mit vollzeitbeschäftigtem Mann und nicht erwerbstätiger Frau, mit der sich etwa die klassische Hausfrauenehe des Ernährermodells erfassen lässt, spielt heute im Osten mit $6 \%$ aller Paarhaushalte eine marginale Rolle (anders als im Westen, wo in jedem fünften Paarhaushalt die Frau nicht beruflich tätig ist). Für beide Landesteile gilt, dass der Anteil der Paare mit nicht erwerbstätiger Hausfrau seit 1990 zurückgegangen ist.

Bei den Zweiverdienerhaushalten lassen sich verschiedene Arbeitszeitkonstellationen unterscheiden, je nachdem, ob beide Partner Vollzeit arbeiten oder die Frau nur teilzeit- bzw. geringfügig beschäftigt ist. Während im Westen Deutschlands sehr viele Frauen Teilzeit arbeiten und damit häufig nur einen Zuverdienst zum Familieneinkommen erarbeiten, spielt diese Konstellation von vollzeitbeschäftigtem Mann und teilzeitbeschäftigter Frau im Osten eine weniger große Rolle. Am häufigsten wird in Ostdeutschland Vollzeitarbeit des Mannes mit Vollzeitarbeit der Frau kombiniert. 41 \% der Paare sind hier Vollzeit-Vollzeit-Paare (in Westdeutschland $23 \%$ ). Zweiverdienerpaare mit teilzeitbeschäftigter Frau machen im Osten $28 \%$ (in Westdeutschland $40 \%$ ) der Paarhaushalte aus (Tabelle 1).

Bemerkenswert ist hierbei die Veränderung im Transformationsprozess: Während die Vollzeit-Vollzeit-Paare von $65 \%$ zu $41 \%$ sehr deutlich abnahmen, nahm spiegelbildlich die Vollzeit-Teilzeit-Konstellation im Osten zu. Allerdings war der Anteil der Vollzeit-Vollzeit-Paare im Jahre 1990 besonders hoch, ${ }^{11}$ im Vergleich zu 1991 fällt die Abnahme weniger drastisch aus (Vollzeit-Vollzeit-Paare 47 \%). ${ }^{12}$ Dennoch kann die Zunahme von Paaren mit teilzeitbeschäftigter Frau, die parallel zur stetig steigenden Teilzeitquote unter ostdeutschen Frauen erfolgte, ein Hinweis auf sich verändernde Geschlechterarrangements im Verlauf des Transformationsprozesses sein. Denn in einer solchen Konstellation ist zweierlei zu vermuten: erstens, dass die Hausarbeit weniger gleichmäßig aufgeteilt ist und die beruflich weniger stark eingebundene Frau den Hauptteil der unbezahlten Arbeit übernimmt (StBA 2004; Kratzer et al. 2005). Und zweitens ist die Annahme plausibel, dass die Einkommen beider Partner sich stärker unterscheiden als bei der Vollzeit-Vollzeit-Konstellation, sodass hier möglicherweise die ökonomische Abhängigkeit der Frauen von ihrem

Tabelle 1: Erwerbskonstellationen 1990, 2000 und 2007 in Paarhaushalten ${ }^{1}$ mit und ohne Kinder - in \% -

\begin{tabular}{|c|c|c|c|c|c|c|c|c|c|}
\hline \multirow[t]{2}{*}{ Erwerbskonstellationen } & 1990 & 2000 & 2007 & 1990 & 2000 & 2007 & 1990 & 2000 & 2007 \\
\hline & \multicolumn{3}{|c|}{ Ostdeutschland } & \multicolumn{3}{|c|}{ Westdeutschland } & \multicolumn{3}{|c|}{ Deutschland gesamt } \\
\hline Beide Vollzeit & 65 & 44 & 41 & 22 & 24 & 23 & 31 & 28 & 26 \\
\hline Mann Vollzeit/Frau Teilzeit & 16 & 14 & 28 & 26 & 30 & 40 & 24 & 27 & 38 \\
\hline $\begin{array}{l}\text { Mann Vollzeit/Frau nicht } \\
\text { erwerbstätig }\end{array}$ & 13 & 9 & 6 & 34 & 26 & 20 & 29 & 23 & 18 \\
\hline $\begin{array}{l}\text { Mann Vollzeit/ } \\
\text { Frau erwerbslos }\end{array}$ & - & 11 & 7 & 2 & 2 & 3 & 2 & 4 & 4 \\
\hline $\begin{array}{l}\text { Mann Teilzeit/Frau Teil- } \\
\text { oder Vollzeit }\end{array}$ & 2 & 1 & 3 & 1 & 2 & 4 & 2 & 2 & 4 \\
\hline $\begin{array}{l}\text { Mann nicht erwerbstätig/ } \\
\text { Frau Teil- oder Vollzeit }\end{array}$ & 4 & 6 & 4 & 8 & 7 & 3 & 7 & 7 & 3 \\
\hline $\begin{array}{l}\text { Mann arbeitslos/Frau Teil- } \\
\text { oder Vollzeit }\end{array}$ & - & 7 & 4 & 2 & 2 & 3 & 1 & 3 & 3 \\
\hline $\begin{array}{l}\text { Beide nicht erwerbstätig } \\
\text { oder arbeitslos }\end{array}$ & 1 & 9 & 6 & 5 & 7 & 3 & 4 & 7 & 4 \\
\hline Restliche Konstellationen & - & - & 1 & - & - & 1 & - & - & 1 \\
\hline $\begin{array}{l}\text { Gesamt } \\
\text { darunter: }\end{array}$ & 100 & 100 & 100 & 100 & 100 & 100 & 100 & 100 & 100 \\
\hline $\begin{array}{l}\text { Zweiverdienerpaare } \\
\text { (Summe Zeile 1, 2, 5) }\end{array}$ & 83 & 59 & 72 & 49 & 56 & 67 & 57 & 57 & 68 \\
\hline $\begin{array}{l}\text { Paare mit einem/einer } \\
\text { arbeitslosen Partner/in } \\
\text { (Summe Zeile 4, 7) }\end{array}$ & - & 18 & 11 & 4 & 4 & 6 & 3 & 7 & 7 \\
\hline $\begin{array}{l}\text { 1) Männer unter } 60 \text { Jahre, Frauen z } \\
\text { von Rundungen. } \\
\text { Quelle: SOEP, Sonderauswertung. }\end{array}$ & en 20 & 55 & Abv & g vol & au & & & TEIL & $N$ \\
\hline
\end{tabular}

Partner gegeben ist. Bevor diese Frage behandelt wird, muss noch auf Erwerbskonstellationen hingewiesen werden, die es 1990 in Ostdeutschland gar nicht gegeben hatte: ein Partner ist erwerbstätig und der oder die andere arbeitslos, oder beide sind nicht erwerbstätig bzw. arbeitslos. In $11 \%$ der Paarhaushalte war 2007 ein Partner Frau oder Mann - arbeitslos (Tabelle 1), bei weiteren $6 \%$ der Paare war weder Mann noch Frau erwerbstätig. ${ }^{13}$

\subsection{EINKOMMENSRELATIONEN}

Zu welchen Einkommensrelationen im Paarzusammenhang führen nun die gezeigten Erwerbskonstellationen? Aus den Untersuchungen von Trappe/Sørensen (2005) wissen wir, dass 1990 der relative Einkommensbeitrag aus Erwerbstätigkeit von ostdeutschen Frauen zum Haushaltsnettoeinkommen $32 \%$ betrug. Dieser ist - trotz der Zunahme von Teilzeitarbeit unter Frauen bis 2002 mit $31 \%$ etwa gleich geblieben. Ihr Einkommensbeitrag lag damit deutlich höher als der von westdeutschen Frauen (20\%). Der relative Einkommensbeitrag von ostdeutschen Männern hat dagegen deutlich abgenommen (von 52\% auf $41 \%$ ). Gestiegen ist dafür der Anteil anderer Einkommensarten aus Sozialtransfers (ebd.).

In der im Rahmen unseres Projektes durchgeführten Auswertung des SOEP wurde entsprechend unserer Fragestellung etwas anders vorgegangen. Wir fragten danach, wer als Haupteinkommensbezieher die Familie „ernährt“, also ob das Familieneinkommen im Wesentlichen auf dem Einkommen des männlichen Haupteinkommensbeziehers beruht, ob es sich in etwa gleichem Maße aus dem Einkommen beider Partner zusammensetzt oder ob die Familie eine weibliche Haupteinkommensbezieherin aufweist. Wir errechneten zum einen die Anteile der Haushalte, in denen jeweils der Mann oder die Frau den Hauptteil des Einkommens erwirtschaftet (mit über $60 \%$ des individuell zurechenbaren Einkommens; haushaltsbezogene Transfers wurden ausgeklammert). Und zum ande-

11 In den Turbulenzen der Umbrüche am Arbeitsmarkt im Jahr 1990 stockten wahrscheinlich sehr viele teilzeitbeschäftigte Frauen, wo es möglich war, ihre Arbeitszeit auf. Der Anteil von VollzeitVollzeit-Paaren dürfte am Ende der DDR bei einer Teilzeitquote von $27 \%$ niedriger gelegen haben (SOEP-Daten liegen für 1989 nicht vor).

12 Vgl. WSI-FrauenDatenReport 2005 (Bothfeld et al. 2005), hier: CD-Romtabelle 3. A.25

13 Paare mit zwei arbeitslosen Partner lassen sich auf Grundlage unserer Auswertung nicht exakt bestimmen, sie sind zusammengefasst mit solchen Paaren, bei denen ein oder beide Partner aus anderen Gründen nicht erwerbstätig sind, weil z.B. erwerbsunfähig, in Ausbildung o.ä. 


\begin{tabular}{|c|c|c|c|c|c|c|}
\hline \multicolumn{7}{|c|}{$\begin{array}{l}\text { Tabelle 2: Paarhaushalte* mit Haupteinkommensbeziehern oder } \\
\text {-bezieherinnen und Paarhaushalte mit egalitärer Einkommens- } \\
\text { erwirtschaftung } 1991 \text { und } 2006 \text { - in \% - }\end{array}$} \\
\hline \multirow{2}{*}{$\begin{array}{l}\text { Das Familien- } \\
\text { einkommen } \\
\text { erwirtschaftet... }\end{array}$} & \multicolumn{3}{|c|}{1991} & \multicolumn{3}{|c|}{2006} \\
\hline & $\begin{array}{l}\text { Ost- } \\
\text { deutschland } \\
n=1.178\end{array}$ & $\begin{array}{l}\text { West- } \\
\text { deutschland } \\
n=1.782\end{array}$ & $\begin{array}{l}\text { Gesamt } \\
\mathrm{n}=\mathbf{2 . 9 6 0}\end{array}$ & $\begin{array}{l}\text { Ost- } \\
\text { deutschland } \\
n=1.316\end{array}$ & $\begin{array}{l}\text { West- } \\
\text { deutschland } \\
n=4.178\end{array}$ & $\begin{array}{l}\text { Gesamt } \\
\mathrm{n}=5.494\end{array}$ \\
\hline $\begin{array}{l}\text {...die Frau zu } \\
\text { mehr als } 60 \%\end{array}$ & 11,2 & 6,9 & 8,6 & 14,8 & 10,6 & 11,6 \\
\hline $\begin{array}{l}\text {...der Mann zu } \\
\text { mehr als } 60 \%\end{array}$ & 44,8 & 69,2 & 59,5 & 40,7 & 61,5 & 56,5 \\
\hline $\begin{array}{l}\text {...beide Partner } \\
\text { etwa gleich zwischen } \\
40 \% \text { und } 60 \%\end{array}$ & 44,0 & 23,9 & 31,9 & 44,5 & 27,9 & 31,9 \\
\hline Insgesamt & 100 & 100 & 100 & 100 & 100 & 100 \\
\hline \multicolumn{7}{|c|}{$\begin{array}{l}\text { *) mit mindestens einer Erwerbsperson unter } 65 \text { Jahren. } \\
\text { Quelle: Sonderauswertung SOEP für das WSI } 2008 .\end{array}$} \\
\hline
\end{tabular}

ren quantifizierten wir die Haushalte, in denen beide etwa gleichgewichtig dazu beitragen (Mann und Frau haben jeweils einen Anteil zwischen $40 \%$ und $60 \%$ am Haushaltseinkommen).

Die Analyse zeigt:

(1) In Paarhaushalten in Ostdeutschland entfiel 2006 mit knapp $45 \%$ der größte Anteil der Paarhaushalte auf einen Typ, bei dem beide Partner in etwa gleichem Maße zum Familieneinkommen beitragen ( $\mathrm{Ta}$ belle 2). Der Anteil dieser Haushalte mit relativ egalitärer Einkommenserwirtschaftung ist im Vergleich zu 1991 praktisch unverändert geblieben.

(2) Paare mit männlichem Haupternährer waren 1991 ebenso häufig wie die mit egaging im Transformationsprozess von ca. $45 \%$ bis auf $41 \%$ (2006) zurück.

(3) Im Gegenzug stieg der Anteil der Paarhaushalte mit weiblicher Haupteinkommensbezieherin von ca. $11 \%$ (1991) auf $15 \%$ (2006).

Damit kommen nicht-traditionale Einkommensrelationen in knapp $60 \%$ der Paarhaushalte vor. Hier ist die Frau von ihrem Partner nicht ökonomisch abhängig. Allerdings kann auch in den Haushalten, in denen die Frau unter $40 \%$ des gemeinsamen Einkommens erwirtschaftet, ihr Anteil substanziell sein, und es liegt - je niedriger das Haushaltseinkommen, umso mehr - eher eine gegenseitige wirtschaft- liche Abhängigkeit beider Partner vor (Trappe/Sørensen 2005).

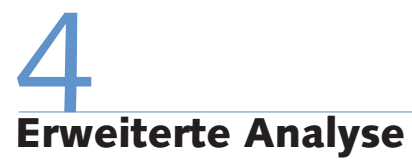

Um die Bedeutung der ökonomischen Unabhängigkeit von Frauen und der Rolle der weiblichen Erwerbseinkommen für die Existenzsicherung von Familien erfassen zu können, haben wir die Analyse um die Haushalte der Alleinerziehenden erweitert. Basis der Auswertungen sind nun alle Mehrpersonenerwerbshaushalte. ${ }^{14}$ Die Analyse der Einkommensstrukturen in Mehrpersonenhaushalten ergibt:
(1) Der Anteil alleinerziehender Frauen, die aus ihrem Einkommen die Familie ernähren, machte 2006 in Ostdeutschland rund $9 \%$ aus und war damit gegenüber 1991 gewachsen (Abbildung 1). Hier versorgt die Frau aus dem Erwerbseinkommen nicht einen Partner, sondern ein Kind oder mehrere Kinder.

\footnotetext{
14 Paarhaushalte mit und ohne Kinder mit mindestens einer Erwerbsperson unter 65 Jahren sowie Alleinerziehendenhaushalte. Ausgeklammert blieben hier Single- und Rentnerhaushalte. Durch die veränderte Berechnungsbasis verändern sich die Anteilswerte gegenüber Tabelle 2 .
}

\section{Abb. 1: Einkommenskonstellation in Mehrpersonenhaushalten ${ }^{1}$ in Ostdeutschland 1991 und 2006}

- Angaben in \% -

1991

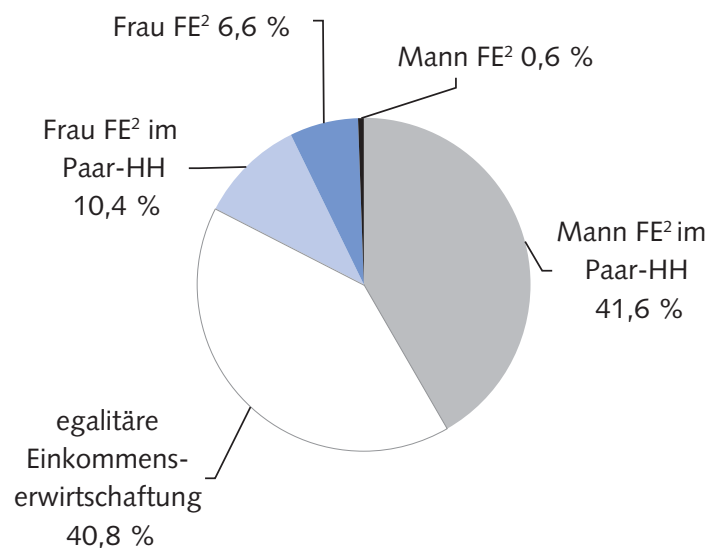

2006

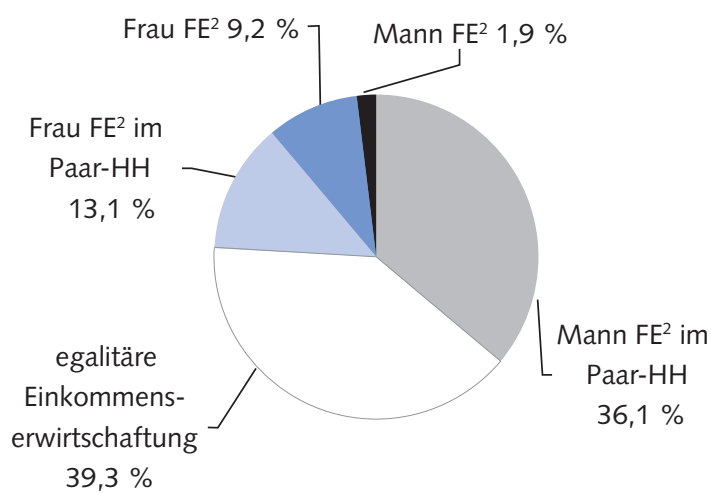

1) Basis sind hier alle Mehrpersonenhaushalte mit mindestens einer Erwerbsperson unter 65 Jahren (Paarhaushalte mit und ohne Kinder sowie Alleinerziehendenhaushalte mit Kindern) 2) $\mathrm{FE}=$ Familienernäherin/Familienernährer. 
(2) Der Anteil der Haushalte, in denen die Frau weniger als $40 \%$ des Einkommens erwirtschaftet und somit „Zuverdienerin“ (oder in seltenen Fällen Hausfrau) ist, macht bei dieser Betrachtung ein reichliches Drittel (36 \%) der Mehrpersonenerwerbshaushalte aus.

(3) Im Zeitverlauf zugenommen hat in Ostdeutschland der Anteil weiblicher Familienernährerinnen (Haupteinkommensbezieherinnen im Paarhaushalt und Alleinerziehende zusammengenommen): von rund $17 \%$ (1991) ist ihr Anteil an den Mehrpersonenerwerbshaushalten auf $22 \%$ (2006) angestiegen. Unter den weiblichen Familienernährerinnen ist rund jede zweite eine Alleinerziehende. ${ }^{15}$

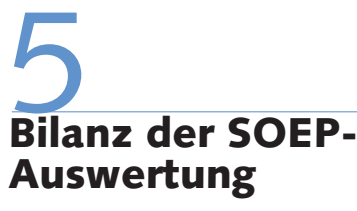

Ausgeklammert waren aus dieser Berechnung die Single-Haushalte. Geht man davon aus, dass auch in diesem Haushaltstyp Frauen wie Männer normalerweise ihren Lebensunterhalt eigenständig sichern, dann zeigt sich, dass für Frauen in Ostdeutschland Erwerbsarbeit für die eigenständige Existenzsicherung grundsätzlich die Regel ist. Und tatsächlich ist in Ostdeutschland nur noch eine Minderheit der Frauen in ihrer Existenzsicherung von ihrem Partner abhängig. Dies hat sich im Laufe des Transformationsprozesses nicht verändert. Allerdings verstellt diese Betrachtungsweise den Blick dafür, dass Frauen sich oder ihre Familie häufig mit sehr niedrigen Einkünften ernähren (Klenner/Klammer 2009). Sie sind im Niedrigeinkommenssektor stark überrepräsentiert (Kalina/Weinkopf 2009), ein Teil muss aufstockende Arbeitslosengeld (ALG)-II-Leistungen beziehen.

Frauen sind heute sogar häufiger Haupteinkommensbezieherinnen als zum Zeitpunkt der deutschen Einheit, wenngleich teilweise mit armutsnahen Einkünften. Die Bedeutung der eigenständigen Einkommenserwirtschaftung durch Frauen wäre noch größer, würde nicht ein Teil der Frauen durch Arbeitslosigkeit oder unfreiwillige Teilzeit und Minijobs daran gehindert, ein eigenes Existenz sicherndes Einkommen zu erarbeiten.

Es zeigt sich: In Ostdeutschland dominiert das Zweiverdienermodell, die Haus- frauenehe hat nur sehr geringe Bedeutung. Paare mit einem egalitären Erwerbsbeitrag beider Geschlechter machten schon 1991 einen hohen Anteil aus, und dieser ist im Transformationsprozess stabil geblieben. Verschiebungen hat es in zweierlei Hinsicht gegeben: Weit weniger Paare kombinieren heute Vollzeitarbeit des Mannes mit Vollzeitarbeit der Frau, dafür ist der Anteil der Paare mit teilzeitbeschäftigter Frau deutlich angestiegen. Zum anderen ist der Anteil weiblicher Zuverdienerinnen und männlicher Ernährer leicht zurückgegangen, der Anteil weiblicher Familienernährerinnen (alleinerziehend oder im Paarhaushalt) hat dagegen zugenommen. Ein Teil der Paare ist daran gehindert, das normativ erwünschte Zweiverdienermodell zu leben, weil nur ein Partner in die Erwerbsarbeit integriert ist.

Die Daten machen deutlich: Ein Wandel hin zu traditionelleren Formen lässt sich auch auf der Paarebene nicht nachweisen, eher das Gegenteil. Jedoch ist die Möglichkeit der eigenständigen Existenzsicherung für Frauen und Männer aufgrund von Arbeitslosigkeit und Niedriglöhnen eingeschränkt.

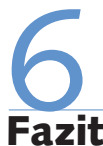

\section{Fazit und offene Fragen}

Auch wenn es einzelne Befunde gibt, deren Auswirkungen auf Geschlechterarrangements und Gleichstellung noch weiter beobachtet werden müssen - wie der wachsende Anteil teilzeitarbeitender Frauen und der höhere Einkommensabstand jüngerer Frauen gegenüber Männern -, ist die Gesamtbilanz von 20 Jahren Transformationsprozess bisher eindeutig: von einem Rückfall in alte Muster, also von einer ReTraditionalisierung der Geschlechterverhältnisse, kann keine Rede sein, und zwar weder bei der Betrachtung volkswirtschaftlicher Größen noch auf der Paarebene. Im Gegenteil: Das männliche Ernährermodell, das bereits zum Zeitpunkt der deutschen Vereinigung eine nachlassende Bindekraft zeigte, ist weiter auf dem Rückzug. Nichtkonventionelle Arbeitszeit- und Einkommensrelationen in Paarhaushalten überwiegen. Nimmt man alleinlebende sowie alleinerziehende Frauen hinzu, dann ist die übergroße Mehrheit der ostdeutschen Frauen wirtschaftlich für sich selber verantwortlich. Allerdings gehören arbeitslose und unfreiwillig teilzeitbeschäftigte Frauen häufig zu denjenigen, die ungewollt vom Partnereinkommen abhängig sind. Ohne diese beiden Formen der Unterbeschäftigung wäre der Anteil nicht traditioneller Konstellationen in Ostdeutschland vermutlich noch größer. Eine besonders unkonventionelle Konstellation ist diejenige mit weiblicher Haupteinkommensbezieherin.

Die durchgeführte statistische Analyse vermag allerdings nicht zu erklären, ob und inwieweit ein qualitativ neues Geschlechterarrangement entsteht, wenn Frauen als Familienernährerinnen fungieren. Dieser Frage, also ob es in den Dimensionen Zeit, Geld, Macht und Teilung der unbezahlten Arbeit zu neuen Beziehungen der Geschlechter kommt, gehen wir im Rahmen des laufenden Forschungsprojektes (vgl. Fußnote 3) nach. Unsere Forschungshypothese ist, dass es keinen eindeutigen $\mathrm{Zu}$ sammenhang zwischen den Einkommensrelationen im Paarzusammenhang und der Hierarchie der Partner gibt.

Auch steht die Antwort noch aus, wie sich die Entwicklung der Geschlechterverhältnisse in Ostdeutschland erklären lässt. ${ }^{16}$ Sie unterscheidet sich nicht nur von der in Westdeutschland (wo immerhin die Entwicklungsrichtung die gleiche ist), sondern auch deutlich von den Entwicklungen in den anderen mittel- und osteuropäischen Ländern, die früher zum staatssozialistischen Block gehörten, die von ähnlichen ideologischen Grundlagen ausgingen und ebenfalls eine Politik der Förderung der Frauenerwerbstätigkeit betrieben. Gleichwohl sind dort Anschauungen und Praxis wesentlich traditioneller (Klenner/Leiber 2009). Anknüpfend an Forschungen zur Erklärung der Frauenerwerbsbeteiligung (Pfau-Effinger 2000) sowie zu Einkommensrelationen im Haushalt (Drago et al. 2005) muss die Suche nach Erklärungsansätzen sich auf mindestens vier Faktorenbündel beziehen: ökonomische Faktoren, sodann wohlfahrtsstaatliche Rahmenbedingungen in ihrem Wechselspiel mit kulturellen Leitbildern, das historische Erbe der DDR sowie der Verlauf der deutschen Einigung.

\footnotetext{
15 Zu allen angeführten Konstellationen finden sich die Vergleichsdaten zu Westdeutschland in Klenner/Klammer 2009

16 Erklärungsansätze finden sich unter anderem bei
} Nickel 1993; Trappe 1995 und Dölling 2005. 


\section{LITERATUR}

Beckmann, P. (2002). Teilzeit zwischen Wunsch und Wirklichkeit, in: Bundesarbeitsblatt 11, S. 13-17

Betzelt, S. (2007): „Gender Regimes“: Ein ertragreiches Konzept für die komparative Forschung. Literaturstudie, Universität Bremen, Zentrum für Sozialpolitik, ZeS-Arbeitspapier 12

Bispinck, R./Dribbusch, H./Öz, F. (2009): Geschlechtsspezifische Lohndifferenzen nach dem Berufsstart und in der ersten Berufsphase. Eine Analyse von Einkommensdaten auf Basis der WSI-Lohnspiegeldatenbank in Deutschland und im europäischen Vergleich, hrsgg. vom BMFSFJ. http://www.bmfsfj.de/bmfsfj/generator/BMFSFJ/Service/Publikationen/ publikationen, did=126186.html

Bothfeld, S./Klammer, U./Klenner, Ch./Leiber, S./Thiel, A./Ziegler, A. (2005): WSI-FrauenDatenReport 2005. Handbuch zur wirtschaftlichen und sozialen Situation von Frauen, Berlin

Budde, G.-F. (1997) (Hrsg.): Frauen arbeiten. Weibliche Erwerbstätigkeit in Ost- und Westdeutschland nach 1945, Göttingen

Commission of the European Communities (EC) (2009): Equality between women and men - 2009, Report from the Commission to the European Parliament, the European Economic and Social Committee and the Committee of the Regions, Brussels

Dölling, I. (2003): Ostdeutsche Geschlechterarrangements in Zeiten des Neoliberalismus, in: Potsdamer Studien zur Frauen- und Geschlechter-

forschung 1, S. 7-32

Dölling, I. (2005): Ostdeutsche Geschlechterarrangements in Zeiten des neoliberalen Gesellschaftsumbaus, in: Schäfer, E. et al. (Hrsg.): Irritation Ostdeutschland. Geschlechterverhältnisse seit der Wende, Münster, S. 16-34

Drago, R./Black, D./Wooden, M. (2005): Female breadwinner families. Their existence, persistence and sources, in: Journal of Sociology 4, S. 343-362

Geißler, R. (1993): Sozialer Umbruch als Modernisierung in: Geißler, R. (Hrsg.): Sozialer Umbruch in Ostdeutschland, Opladen, S. 63-91 Gensior, S. (Hrsg.) (1995): Vergesellschaftung und Frauenerwerbsarbeit. Ost-West-Vergleiche, Berlin

Gille, M./Marbach, J. (2004): Arbeitsteilung von Paaren und ihre Belastung mit Zeitstress, in: Statistisches Bundesamt: Alltag in Deutschland. Analysen zur Zeitverwendung, in: Forum der Bundesstatistik, Bd. 43,

S. 86-113

Kalina, Th./Weinkopf, C. (2009): Niedriglohnbeschäftigung weiter gestiegen - zunehmende Bedeutung von Niedriglöhnen, in: IAQ-Report 2009-05

Klammer, U./Klenner, Ch./Ochs, C./Radke, P./Ziegler, A. (2000): WSI FrauenDatenReport, Berlin

Klenner, Ch./Hašková, H. (2009): Variationen des Zweiverdienermodells: Müttererwerbstätigkeit im tschechisch-deutschen Vergleich, in: Klenner, Ch./Leiber, S. (2009) (Hrsg.): Wohlfahrtsstaaten und Geschlechterungleichheit in Mittel- und Osteuropa. Kontinuität und postsozialistische Transformation in den EU-Mitgliedsstaaten, Wiesbaden, S. 193-228 Klenner, Ch./Klammer, U. (2009): Weibliche Familienernährerinnen in West und Ostdeutschland - Wunschmodell oder neue Prekarität?, in: Dokumentation der BMFSFJ-Tagung in der Villa Vigoni im Oktober 2008, (im Erscheinen)

Klenner, Ch./Leiber, S. (Hrsg.) (2009): Wohlfahrtsstaaten und Geschlechterungleichheit in Mittel- und Osteuropa. Kontinuität und postsozialistische Transformation in den EU-Mitgliedsstaaten, Wiesbaden
Kolinsky, E. (2003): Gender and the Limits of Equality, in: Kolinsky, E./ Nickel, H. M. (Hrsg.): Reinventing Gender. Women in Eastern Germany since Unification, London, S. 100-127

Kolinsky, E. /Nickel, H. M. (Hrsg.) (2003): Reinventing Gender. Women in Eastern Germany since unification, London

Kratzer, N./Fuchs, T./Wagner, A./Sauer, D. (2005): Zeitmuster - Zeitverwendung im Kontext von Erwerbsarbeit und Haushalt, in: Soziologisches Forschungsinstitut (SOFI), Institut für Arbeitsmarkt- und Berufsforschung (IAB), Institut für Sozialwissenschaftliche Forschung (ISF), Internationales Institut für empirische Sozialökonomie (INIFES) (Hrsg.): Berichterstattung zur sozio-ökonomischen Entwicklung in Deutschland - Arbeit und Lebensweisen, Erster Bericht, Wiesbaden, S. 381-402

Kröhnert, S./Klingholz, R. (2007): Studie "Not am Mann - Von Helden der Arbeit zur neuen Unterschicht?“ , hrsg. vom Berlin-Institut für Bevölkerung und Entwicklung

Leitner, S./Ostner, I./Schratzenstaller, M. (Hrsg.) (2004): Wohlfahrtsstaat und Geschlechterverhältnis im Umbruch. Was kommt nach dem Ernährermodell?, Jahrbuch für Europa- und Nordamerika-Studien 7, Wiesbaden Lewis, J. (2004): Auf dem Weg zur "Zwei-Erwerbstätigen“-Familie, in: Leitner, S. et al. (2004): Wohlfahrtsstaat und Geschlechterverhältnis im Umbruch. Was kommt nach dem Ernährermodell?, Jahrbuch für Europaund Nordamerika-Studien 7, Wiesbaden, S. 62-84

Nickel. H. M. (1993): Mitgestalterinnen des Sozialismus - Frauenarbeit in der DDR, in: Helwig, G./Nickel, H. M. (1993): Frauen in Deutschland 1945-1992, Berlin, S. 233-256

Pfau-Effinger, B. (1998): Arbeitsmarkt- und Familiendynamik in Europa Theoretische Grundlagen der vergleichenden Analyse, in: Geissler, B./ Maier, F./Pfau-Effinger, B. (1998): FrauenArbeitsMarkt. Der Beitrag der Frauenforschung zur sozio-ökonomischen Theorieentwicklung, Berlin, S. 177-194

Pfau-Effinger, B. (2000): Kultur und Frauenerwerbstätigkeit in Europa. Theorie und Empirie des internationalen Vergleichs, Opladen Rohnstock, K. (Hrsg.) (1994): Stiefschwestern. Was Ost-Frauen und West-Frauen voneinander denken, Frankfurt a. $M$.

Schäfer, E./Dietzsch, I./Drauschke, P./Peinl, I./Penrose, V./Scholz, S./ Völker, S. (Hrsg.) (2005): Irritation Ostdeutschland. Geschlechterverhältnisse seit der Wende, Münster

Schenk, S. (2003): Employment opportunities and labour market exklusion: towards a new pattern of gender stratification?, in: Kolinsky, E./ Nickel, H. M. (Hrsg.): Reinventing Gender. Women in Eastern Germany since Unification, S. 53-77

Schmidtke, H. (2004): Frauen in den neuen Bundesländern, in: Winkler, G.(Hrsg.): Sozialreport 2004. Daten und Fakten zur sozialen Lange in den neuen Bundesländern, Berlin, S. 278-299

Scholz, S. (2005): „Der Mann, der großer Ernährer der Familie, das ist in den Köpfen nicht so drin“ - Identitätskonstruktionen ostdeutscher Männer, in: Schäfer, E. et al.: Irritation Ostdeutschland. Geschlechterverhältnisse seit der Wende, Münster, S. 135-146 Statistisches Bundesamt (StBA) (2004): Alltag in Deutschland. Analysen zur Zeitverwendung, in: Forum der Bundesstatistik, Bd. 43 Statistisches Bundesamt (StBA) (Hrsg.) (2006): Datenreport 2006, Bundeszentrale für politische Bildung Bonn

Statistisches Bundesamt (StBA) (2009a): Pressemitteilung des Statistischen Bundesamtes 160 vom 28.04 
Statistisches Bundesamt (StBA) (2009b): Mikrozensus Arbeitstabellen Statistisches Bundesamt (StBA) (2009c): Niedrigeinkommen und Erwerbstätigkeit. Begleitmaterial zum Pressegespräch am 19. August Statistisches Bundesamt (StBA) (2009d): Sondertabellen zu Lebensgeborenenstatistik http://www.destatis.de/jetspeed/portal/cms/Sites/ destatis/Internet/DE/Presse/pk/2009/Erwerbstaetigkeit/begleitheft_ Erwerbstaetigkeit, property=file.pdf (am 20.8.2009)

Thelen, T./Baerwolf, A.( 2007): Traditionalisierung in der Flexibilisierung Familiäre Arbeitsteilung in Ostdeutschland, in: Szydlak, M. (Hrsg.): Flexibilisierung. Folgen für Arbeit und Familie, S. 275-294

Trappe, H. (1995): Emanzipation oder Zwang? Frauen in der DDR zwischen Beruf, Familie und Sozialpolitik, Berlin
Trappe, H./Sørensen, A. (2005): Economic Relations between Women and Their Partners: An East and West German Comparison after Reunification, in: DIW-Discussion paper 350, Berlin

Völker, S. (2004): Hybride Geschlechterpraktiken. Erwerbsorientierungen und Lebensarrangements von Frauen im ostdeutschen Transformationsprozess, Wiesbaden

Wagner, A. unter Mitarbeit von Dathe, D./Fuchs, T./Stolz-Willig, B./ Botsch, E. (2006): Kontinuität trotz Transformation? Normalarbeitsverhältnis und geschlechtsspezifische Arbeitsteilung in Ostdeutschland, Abschlussbericht des Projektes der Hans-Böckler-Stiftung 